\title{
Fading-loss assessment in atmospheric free- space optical communication links with on-off keying
}

\author{
Dirk Giggenbach, MEMBER SPIE \\ Hennes Henniger \\ German Aerospace Center (DLR) \\ Institute of Communication and Navigation \\ Muenchnerstrasse 20 \\ 82230 Wessling, Germany \\ E-mail: dirk.giggenbach@dlr.de
}

\begin{abstract}
Link-budget calculations are a common way to assess system parameters, such as the required transmitter power and receiver sensitivity, in free-space optical (FSO) communication systems. One of the biggest challenges for long-range FSO deployment is its signal propagation under turbulent atmospheric conditions, which produce intensity fluctuations. Methods to estimate atmospheric-fading loss in radio-frequency systems cannot be adapted to the FSO channel. Until now no general closed-form methods have been developed to describe the fading loss in such a channel. A method to calculate the losses due to scintillation fading in the threshold approach, based on lognormal statistics of the received power, is presented. (c) 2008 Society of Photo-Optical Instrumentation Engineers. [DOI: 10.1117/1.2903095]
\end{abstract}

Subject terms: fading loss; free-space optics; link budget; aperture averaging.

Paper 070815R received Sep. 28, 2007; revised manuscript received Nov. 16, 2007; accepted for publication Dec. 4, 2007; published online Apr. 25, 2008.

\section{Introduction}

Whenever an optical beam with a longitudinal coherence length of at least several wavelengths passes through atmospheric optical index-of-refraction turbulence (IRT), the distortions imposed on its wavefront will produce random interference patterns in the far field with lateral intensity coherence length $\rho_{I}$, so-called intensity speckle patterns.

When the diameter of the receiver aperture, $D_{\mathrm{Rx}}$, is larger than $\rho_{I}$, aperture averaging of the received intensity distribution takes place. This effect reduces the strength of received power fluctuations below the power fluctuations observed by a point receiver, and thus improves system performance of an optical communication link. A parameter for the strength of intensity fluctuations is the normalized variance of the intensity-usually called the intensity scintillation index $\sigma_{I}^{2}$-and for the received power fluctuations the power scintillation index $\sigma_{P}^{2}$ is used.

Here a straightforward method to calculate the corresponding scintillation-fading loss $a_{\text {sci }}$ (in decibels) is developed, depending on $\sigma_{P}^{2}$ and the probability $p_{\text {thr }}$ that the received power falls below the limit $P_{\min }\left(p_{\text {thr }}\right.$ equals the fraction of link outage time). The basic idea for this approach was presented by Yura and McKinley, ${ }^{1}$ and the method is directly applicable for performance evaluations of systems with on-off keying (OOK). Only IRT-induced fading including aperture averaging is investigated here. One should consider that mispointing of the transmit beam and tracking errors of the receiver will generate additional signal fading.

The derivation of $\sigma_{P}^{2}$ from $\sigma_{I}^{2}, \rho_{I}$, and $D_{\mathrm{Rx}}$ is based on established approximation methods as stated in Ref. 2. The evaluation of aperture averaging is based on the experimentally proven assumption of a lognormal density function of

0091-3286/2008/\$25.00 @ 2008 SPIE both received intensity and collected power. Also, more thorough investigations of this effect approve the use of a lognormal-like approximation for the receiver $(\mathrm{Rx})$ power distribution even when the intensity distribution in a strongturbulence scenario no longer follows lognormal behavior.

\section{System Model of IRT-Disturbed Optical Free-Space Communications}

The quality of the received signal is determined by the different noise sources (either signal-dependent or nonsignal-dependent) and the received signal power $P_{\mathrm{Rx}}$ itself. A certain short-term (mean over 1 and 0 bit states) minimum received power $P_{\min }$ can be defined, below which the receiver no longer satisfies a system-inherent quality standard, which is usually given by a maximum bit error probability. When 1's and 0's are equally distributed in an OOK data stream, $P_{\min }$ can be directly measured with a power meter, and it satisfies $P_{\min }=\frac{1}{2} P_{\text {min_1 } 1}$. Here $P_{\text {min_1 }}$ denotes the minimum allowed Rx power during reception of a logical 1. The exact value of $P_{\min }$ depends on several technical constraints such as the background light and the performance of the receiver electronics, and in practice should be determined in a measurement setup.

Time spectra are not covered with this approach and are not investigated here. But as a rough indication it should be mentioned that atmospheric IRT typically reduces the channel coherence time $\tau_{c}$ to values between 50 and $2 \mathrm{~ms}$ in applications with fixed terminals, where the time spectrum is governed by the orthogonal wind speed. In scenarios with moving partners such as aircraft or satellites, $\tau_{c}$ can be as low as $0.2 \mathrm{~ms}^{3}{ }^{3}$ With the usually very high data rates in optical communications (from several megabits per second up to some gigabits per second), this still implies extremely slow fading, for the ratio of channel coherence time to bit duration is at least $10^{4}$. This is hard to cope with by conventional forward error correction, for the required coder 
memory would be in the range of millions of bits. One solution here is the implementation of very long interleavers or packet-layer coding, ${ }^{4}$ both providing the necessary long constraint lengths. In this paper we treat system performance without these techniques.

\subsection{Statistical Parameters for Intensity Scintillations}

Because the intensity field is an ergodic process in time and space, its normalized variance-called the intensity scintillation index $\sigma_{I}^{2}$-at link distance $L$ can be calculated from spatial or temporal intensity statistics, using transverse coordinates $x$ and $y$ and time $t$ :

$$
\begin{aligned}
\sigma_{I}^{2} & =\frac{\left\langle I^{2}(L, x, y, t)\right\rangle-\langle I(L, x, y, t)\rangle^{2}}{\langle I(L, x, y, t)\rangle^{2}}=\frac{\left\langle I^{2}(L, x, y, t)\right\rangle_{x, y}}{\langle I(L, x, y, t)\rangle_{x, y}^{2}}-1 \\
& =\frac{\left\langle I^{2}(L, x, y, t)\right\rangle_{t}}{\langle I(L, x, y, t)\rangle_{t}^{2}}-1 .
\end{aligned}
$$

The scintillation index $\sigma_{I}^{2}$ is usually evaluated in terms of the Rytov variance $\sigma_{R}^{2}$, which is an analytical measure for the integrated amount of turbulence along the link path, weighted with the wavelength $\lambda$ :

$$
\begin{aligned}
& \sigma_{R}^{2}=2.25 k^{7 / 6} \int_{0}^{L} C_{n}^{2}(z) \cdot(L-z)^{5 / 6} \mathrm{~d} z \\
& \begin{aligned}
C_{n}^{2} & =\text { const } \\
& =1.23 C_{n}^{2} k^{7 / 6} L^{11 / 6}, \quad k
\end{aligned}=\frac{2 \pi}{\lambda} .
\end{aligned}
$$

For weak turbulence (i.e., $\left.\sigma_{R}^{2}<0.5\right), \sigma_{I}^{2}$ is nearly equal to $\sigma_{R}^{2}$, whilst for $\sigma_{R}^{2}>0.5, \sigma_{\mathrm{I}}^{2}$ grows more slowly (intermediate turbulence), and after a maximum at typically $\sigma_{R}^{2}$ $\approx 2 . .8$ (strong turbulence) drops and asymptotically tends towards unity (saturation regime). ${ }^{2}$ Therefore, the most uncertain region for predicting $\sigma_{I}^{2}$ is around its peak.

Analytical calculation of $\sigma_{I}^{2}$ based on locationdependent turbulence parameters along the link path-such as $C_{n}^{2}$ (index-of-refraction structure constant), $l_{0}$ (inner scale of turbulence), and $L_{0}$ (outer scale of turbulence)and on the transmit beam profile (plane, spherical, Gaussian, flat-top, etc.) is often limited to certain regimes of turbulence strength. Recently, a more general analytical description has been given, ${ }^{2}$ which allows the calculation of both $\sigma_{I}^{2}$ and $\sigma_{P}^{2}$ for some more general scenarios from weak through strong to saturated turbulence. Especially plane and spherical waves are treated sufficiently to allow the calculation of $\sigma_{P}^{2}$ for practical scenarios.

The spherical or plane wave is always only an approximation to the practical case, where either cut Gaussian beams or multimode profiles are transmitted. As a rule of thumb, the plane wave produces stronger scintillations than spherical waves, while Gaussian beams lie somewhere in between, showing low scintillation on axis but sometimes producing extreme variations off axis (see Ref. 2, Figs. 8.1 through 8.6). Especially with very narrow collimated Gaussian beams, the scintillation can increase extremely through mispointing, such as is produced by IRT-induced beam wander. ${ }^{5}$ The peak value of $\sigma_{I}^{2}$ is higher with a larger inner scale of turbulence $l_{0}$.
In mobile inner-atmospheric communication links we are usually dealing with spherical waves, because the transmit-beam divergence has to be widened above the diffraction-limited value to ensure illumination of the partner terminal from an unstable moving platform. Spherical waves are therefore used for the examples here, but the theory presented is not limited to these kinds of waves.

The classification of the transmitted beam as a spherical wave holds when the full $1 / e^{2}$ divergence angle in radians is larger than $7.1(\lambda / L)^{1 / 2}$, as can be deduced from the theory given in Ref. 6 , page 180 , by using an arbitrarily chosen classification limit.

\subsection{Rx-Power Scintillations with Aperture Averaging}

The electrical signal amplitude $S_{\mathrm{el}}(t)$ in standard OOK receivers is proportional to the received optical power $P_{\mathrm{Rx}}(t)$ (neglecting some minor nonlinear effects), which again is the time-dependent integral of the optical intensity $I(x, y, t)$ over the receiver aperture area $A_{\mathrm{Rx}}$. For all further calculations we can therefore use the received optical power $P_{\mathrm{Rx}}$ instead of the electrical amplitude after the receiver front end:

$P_{\mathrm{Rx}}(t)=\iint_{A_{\mathrm{Rx}}} I(x, y, t) \mathrm{d} x \mathrm{~d} y \propto S_{\mathrm{el}}(t)$.

We are here dealing with long-range communication links, where the optical signal distribution at the Rx plane is at least several times larger than the Rx aperture.

While the intensity distribution according to Rytov theory follows lognormal behavior only in weak to intermediate turbulence, numerical and experimental verifications show that lognormal behavior of the received power also applies to a good approximation in all turbulence cases (weak, intermediate, strong, saturation) except when extreme amounts of aperture averaging take place. ${ }^{7-9}$

The ratio between the normalized variance of the received power $\sigma_{P}^{2}$ and that of the intensity field $\sigma_{I}^{2}$ is called the aperture averaging factor $f_{\mathrm{AA}}$ :

$f_{\mathrm{AA}}=\frac{\sigma_{P}^{2}}{\sigma_{I}^{2}}, \quad 0<f_{\mathrm{AA}}<1$.

The aperture-averaging effect acts as a lowpass filter in both the spatial and temporal domains. Assuming a hardrim circular receiver aperture, Ref. 2 gives general approximation formulas for calculating $\sigma_{P}^{2}$ with plane and spherical waves and formulas for the Gaussian beam, which are restricted with respect to the IRT spectrum (subject to innerand outer-scale limits). Note that $\sigma_{P}^{2}\left(\sigma_{R}^{2}\right)$ behaves analogously to $\sigma_{I}^{2}\left(\sigma_{R}^{2}\right)$. However, $\sigma_{P}^{2}\left(\sigma_{R}^{2}\right)$ saturates at values less than unity (Ref. 10, p. 177). Figure 1 illustrates this behavior of $\sigma_{I}^{2}\left(\sigma_{R}^{2}\right)$ with different wave types. Note that a longer wavelength causes larger speckle patterns and thus a reduced aperture-averaging effect, which leads to stronger power scintillations when plotted versus the Rytov index. A rough estimate for the speckle size is $\rho_{I} \approx 0.4 \sqrt{L \lambda}$. 


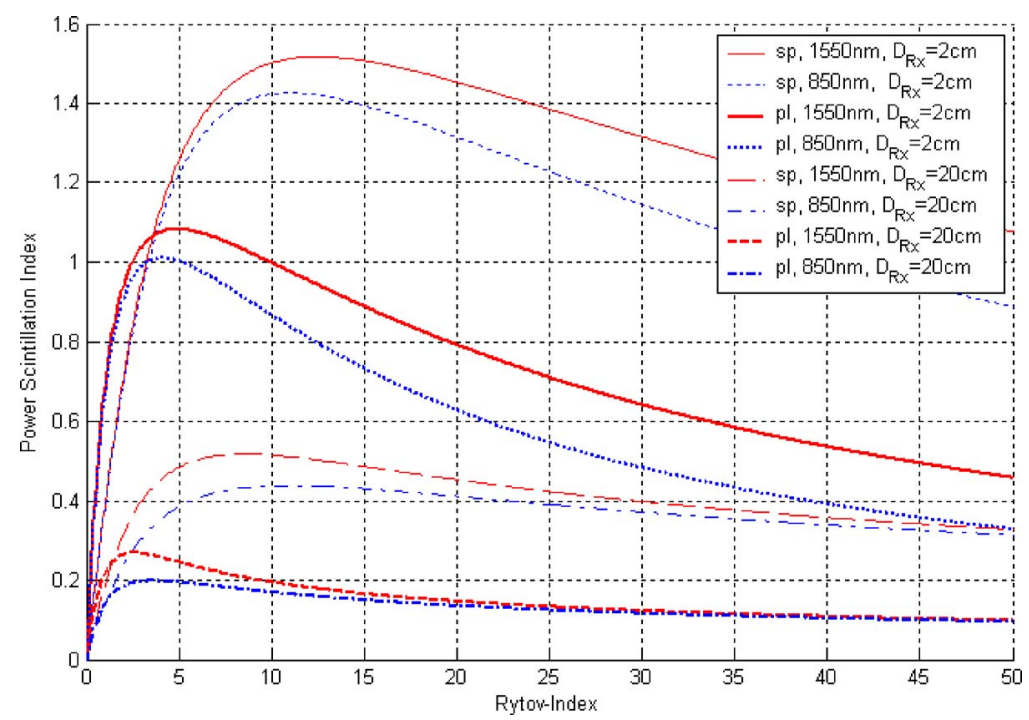

Fig. 1 Examples of power scintillation index $\sigma_{P}^{2}$ versus Rytov index $\sigma_{R}^{2}$ for Kolmogorov spectrum (no inner- and outer-scale bounds) for spherical (sp) and plane (pl) waves, two different wavelengths, and apertures $D_{\mathrm{Rx}}$. Link length is $20 \mathrm{~km}$. Calculated with formulas found in Ref. 2.

\subsection{Lognormal Probability Density Function of $R x$ Power}

The distribution of the Rx power over its whole lateral extent requires power-conservation, because IRT scintillation is a lossless process. Therefore, the basic parameters of its lognormal distribution, $\sigma$ and $\mu$ (the variance and mean of the originating normal distribution), must follow the relation

$\mu=-\frac{1}{2} \ln \left(\sigma_{P}^{2}+1\right)=-\frac{1}{2} \sigma^{2}$

With Eq. (5) we can formulate the lognormal Rx power distribution $p_{p}\left(P_{\mathrm{Rx}}\right)$ for a long-range static link with the long-term average received power $P_{0}$ :

$$
\begin{aligned}
p_{P}\left(P_{\mathrm{Rx}}\right)= & \frac{1}{P_{\mathrm{Rx}}\left[2 \pi \ln \left(\sigma_{P}^{2}+1\right)\right]^{1 / 2}} \\
& \times \exp \left\{-\frac{\left[\ln \left(P_{\mathrm{Rx}} / P_{0}\right)+\frac{1}{2} \ln \left(\sigma_{P}^{2}+1\right)\right]^{2}}{2 \ln \left(\sigma_{P}^{2}+1\right)}\right\} .
\end{aligned}
$$

\section{Receiver Performance Evaluation Using the Threshold Approach}

When $P_{\mathrm{Rx}}$ is fading as described by Eq. (6), a certain acceptable fraction of outage time is defined, during which $P_{\mathrm{Rx}}<P_{\text {min }}$. Then the required power margin between the average reception power $P_{0}$ and $P_{\min }$ must be regarded as an additional loss in the link-budget calculation. This quantity is defined as the scintillation loss $a_{\text {sci }}$ of the transmission system (in decibels):

$a_{\mathrm{sci}}=10 \log _{10}\left(\frac{P_{\min }}{P_{0}}\right), \quad a_{\mathrm{sci}}<0$.

With this threshold approach it is assumed that during times with $P_{\mathrm{Rx}}$ below $P_{\min }$ no data reception is possible at all. This reflects a good-bad-state channel modeling and does not require a detailed investigation of the specific receiver performance; the latter would again depend on modulation format and individual implementation performance. This is of course a rather pessimistic assumption, but it holds quite well with common transmission protocols that show a steep effective bit error ratio (BER) limit—such as Ethernet or digital video broadcast. The fraction of outage time equals the probability $p_{\text {thr }}$ that the actual power falls below $P_{\min }$. This value $p_{\text {thr }}$ can be calculated with the distribution function based on Eq. (6) by using the analogy in Ref. 6, p. 242:

$p_{\text {thr }}\left(P_{\mathrm{Rx}}<P_{\min }\right)=\frac{1}{2}\left(1+\operatorname{erf}\left\{\frac{\ln \left[\frac{P_{\min }}{P_{0}}\left(\sigma_{P}^{2}+1\right)^{1 / 2}\right]}{\left[2 \ln \left(\sigma_{P}^{2}+1\right)\right]^{1 / 2}}\right\}\right)$.

This equation can be found in Ref. 1, where statistics for a point receiver are assumed, but here we extend its validity to power statistics in the presence of aperture averaging. To calculate $a_{\text {sci }}$, Eq. (8) must be solved for $P_{\text {min }}$ :

$\frac{P_{\min }\left(p_{\mathrm{thr}}\right)}{P_{0}}=\frac{\exp \left\{\operatorname{erf}^{-1}\left(2 p_{\mathrm{thr}}-1\right) \cdot\left[2 \ln \left(\sigma_{p}^{2}+1\right)\right]^{1 / 2}\right\}}{\left(\sigma_{P}^{2}+1\right)^{1 / 2}}$.

Then

$$
\begin{aligned}
a_{\text {sci }}= & 4.343\left\{\operatorname{erf}^{-1}\left(2 p_{\text {thr }}-1\right) \cdot\left[2 \ln \left(\sigma_{P}^{2}+1\right)\right]^{1 / 2}\right. \\
& \left.-\frac{1}{2} \ln \left(\sigma_{P}^{2}+1\right)\right\} .
\end{aligned}
$$

As can be seen from Fig. 2, the scintillation loss can easily exceed $20 \mathrm{~dB}$ with typical link requirements (e.g., $\mathrm{BER}<10^{-6}$ ). On the other hand, with saturation and aperture averaging the value will hardly exceed $15 \mathrm{~dB}$, for $\sigma_{P}^{2}$ will then be less then unity. 


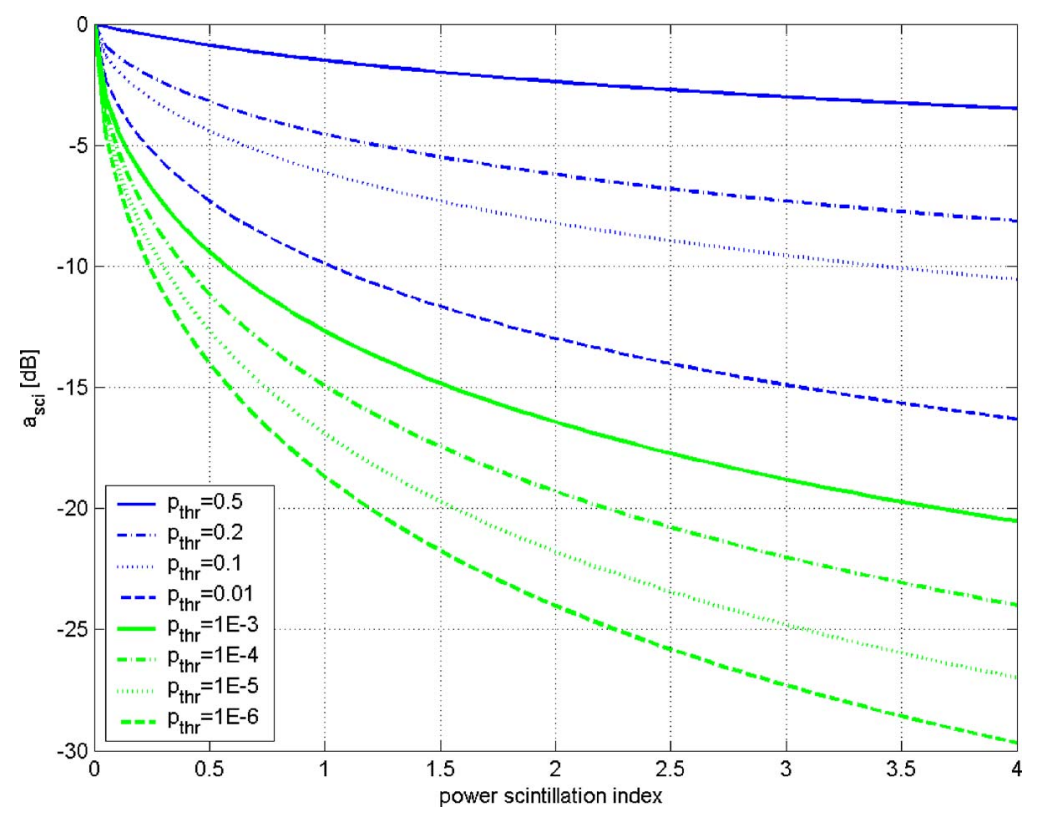

Fig. 2 Scintillation loss $a_{\text {sci }}$ versus power scintillation index $\sigma_{P}^{2}$ with the threshold $p_{\text {thr }}$ as parameter.

Equation (10) can be approximated with an error below $1.1 \mathrm{~dB}$ for $\sigma_{P}^{2}<3$ and $0.7>p_{\text {thr }}>2 \times 10^{-7}$ by the following curve fit:

$a_{\mathrm{sci}} \approx\left[3.3-5.77\left(-\ln p_{\mathrm{thr}}\right)^{1 / 2}\right] \cdot\left(\sigma_{P}^{2}\right)^{0.4}$.

On combining Eqs. (10) and (2) and using Eq. (77) of Chap. 10 in Ref. 2 [which is repeated below as Eq. (12) with substitution for the spherical-wave Rytov variance $\beta_{0}^{2}$ as $\beta_{0}^{2}=0.41 \sigma_{R}^{2}$, for compliance with Eq. (2)] for calculating $\sigma_{P}^{2}$ of a spherical wave, then the scintillation loss for dif- ferent distances, wavelengths, and $D_{\mathrm{Rx}}$ can be evaluated as shown in Figs. 3 and 4:

$$
\begin{aligned}
\sigma_{P, \mathrm{sph}}^{2}\left(D_{\mathrm{Rx}}\right)= & \exp \left\{\frac{0.20 \sigma_{R}^{2}}{\left[1+0.18 d^{2}+0.20\left(\sigma_{R}^{2}\right)^{6 / 5}\right]^{7 / 6}}\right. \\
& \left.+\frac{0.21 \sigma_{R}^{2}\left[1+0.24\left(\sigma_{R}^{2}\right)^{6 / 5}\right]^{-5 / 6}}{1+0.90 d^{2}+0.21 d^{2}\left(\sigma_{R}^{2}\right)^{6 / 5}}\right\}-1
\end{aligned}
$$

with $d=D_{\mathrm{Rx}}(\pi / 2 \lambda L)^{1 / 2}$.

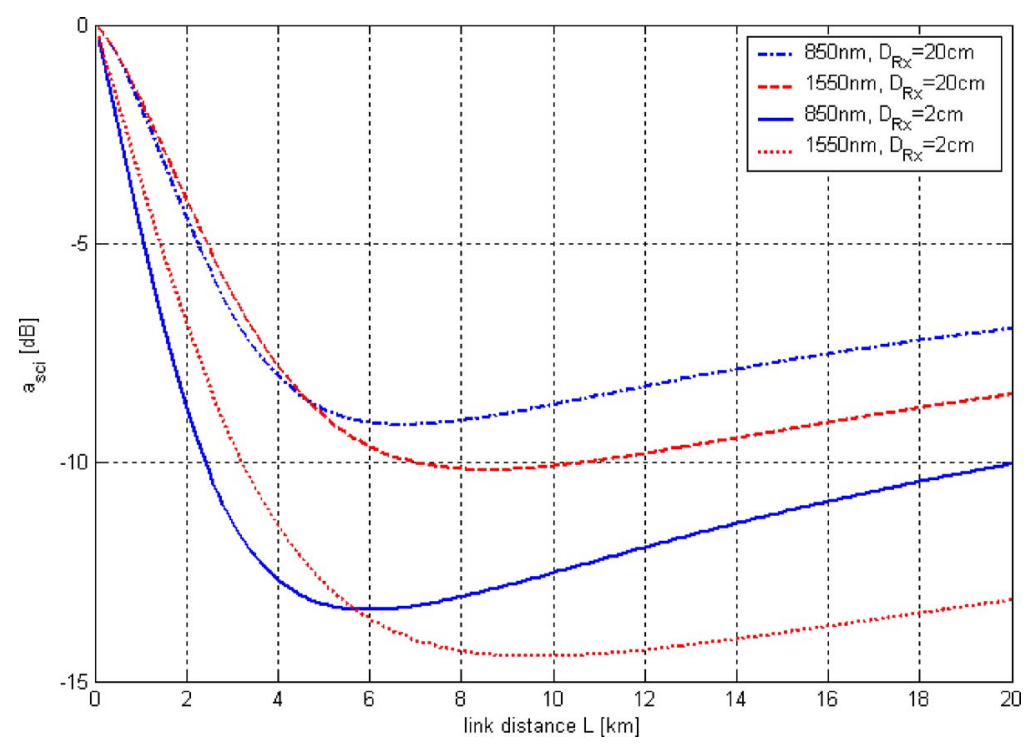

Fig. 3 Scintillation loss $a_{\text {sci }}$ depending on link distance $L$ assuming a spherical wave, with the $R x$ aperture $D_{\mathrm{Rx}}(2$ or $20 \mathrm{~cm})$ and the wavelength $\lambda(850$ and $1550 \mathrm{~nm})$ as parameters. Here the IRT strength is given by a constant $C_{n}^{2}=10^{-14} \mathrm{~m}^{-2 / 3}$, as is suitable for strong near-ground IRT. The fraction of outage time is rather small with $p_{\text {thr }}=0.001$. 


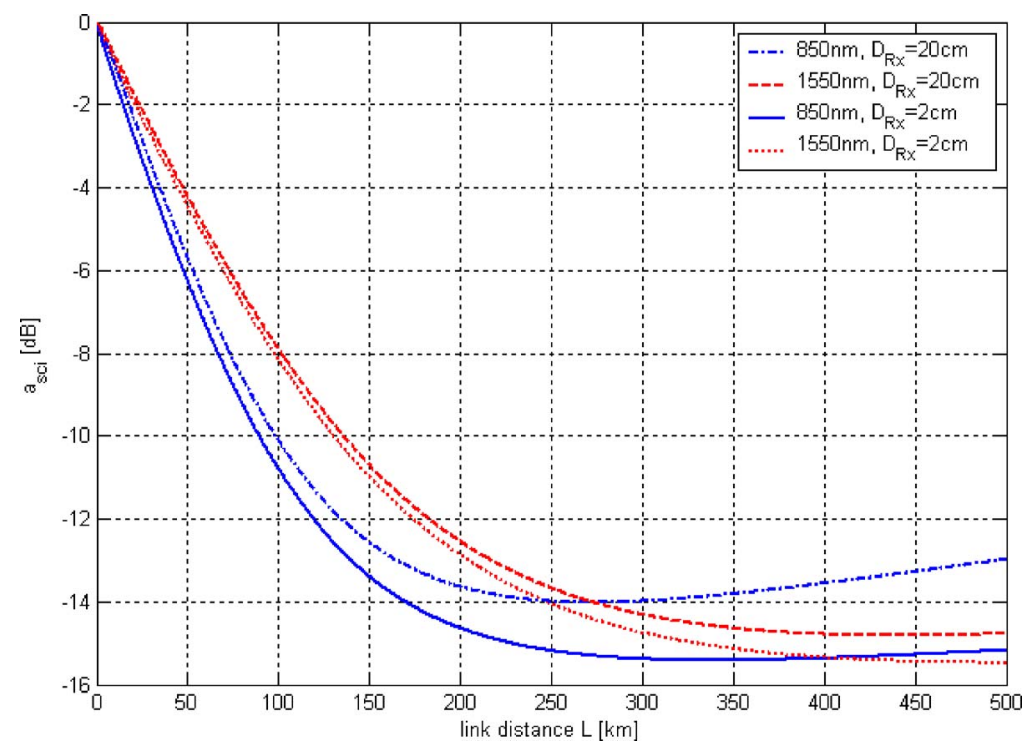

Fig. 4 Same as Fig. 3, but for a constant $C_{n}^{2}=10^{-17} \mathrm{~m}^{-2 / 3}$, as can be observed at higher altitudes (more than $2000 \mathrm{~m}$ above ground).

In Fig. 3 one can observe beneficial behavior of the longer wavelength inside the weak-turbulence regime, while towards longer distances the shorter wavelength is advantageous beyond the PSI peak, due to stronger aperture averaging (caused by smaller speckle sizes, because speckle size scales with wavelength).

In Fig. 4 we observe that at low turbulence strengths together with long propagation distances, the apertureaveraging effect is negligible, therefore small and large apertures behave similarly. Because in this scenario scintillation saturation is not completely reached, the longer wavelength stays advantageous over a long distance range (up to $400 \mathrm{~km}$ in this example).

\section{Conclusion}

In atmospheric optical free-space communication links, the scintillation index and intensity correlation length (together with the aperture diameter of the receiver) are required in order to assess the quality of the received power scintillations. When a fraction of complete data loss is assumed (threshold approach), the scintillation loss can be calculated according to Eq. (10). By the assumption of lognormal power scintillation after aperture averaging, this formula is valid for general scenarios with power-scintillation indices given by appropriate formulas that can be found elsewhere.

The presented approach is not limited to plane or spherical waves, but can be applied to other beam profiles whenever $\sigma_{P}^{2}$ is known.

It is crucial to note that the assumption of synchronous BER measurements for channel estimation assumes stable clock and frame synchronization even after deep fades that have caused a loss of clock. In practice the resynchronization of the bit stream requires some time during which large numbers of data are lost, which again causes a strong increase in the mean BER. Very robust and fault-tolerant clock-recovery technologies would be needed here. Another-more practical-solution is the use of datagram- based asynchronous transmission, where each block comes with its own synchronization preamble, as, e.g., in 100Base-T Ethernet.

Outlook towards coherent systems: Concerning other detection schemes like coherent detection (heterodyning with a local oscillator), the effect of the optical propagation through atmospheric IRT can usually be split up into the effect of field-amplitude scintillations and the reduction of the normalized heterodyning efficiency, each with its own fading distribution, and their mutual correlation. While the Rx power distribution has a constant mean, the distribution of the heterodyning efficiency has a reduced mean due to IRT. Appropriate combination of both distributions together with the corresponding model of the heterodyne receiver would allow the calculation of an overall fading loss also for coherent systems. ${ }^{11}$

\section{References}

1. H. T. Yura and W. G. McKinley, "Optical scintillation statistics for IR ground-to-space laser communication systems," Appl. Opt. 22, 3353 $3358(1983)$

2. L. C. Andrews and R. L. Phillips, Laser Beam Propagation through Random Media, 2nd ed., SPIE Press, Bellingham, WA (2005).

3. N. Perlot, M. Knapek, D. Giggenbach, J. Horwath, M. Brechtelsbauer, Y. Takayama, and T. Jono, "Results of the optical downlink experiment KIODO from OICETS satellite to optical ground station oberpfaffenhofen (OGS-OP)," Proc. SPIE 6457, 645704 (2007).

4. H. Henniger, "Packet-layer forward error correction coding for fading mitigation," Proc. SPIE 6304, 630419 (2006).

5. N. Perlot, D. Giggenbach, H. Henniger, J. Horwath, M. Knapek, and K. Zettl, "Measurements of the beam-wave fluctuations over a 142-km atmospheric path," Proc. SPIE 6304, 630410 (2006).

6. L. C. Andrews and R. L. Phillips, Laser Beam Propagation through Random Media, SPIE Press, Bellingham, WA (1998).

7. N. Perlot, D. Giggenbach, H. Bischl, and F. David, "Discussion of direct detection Rx-power statistics as derived from intensity distributions and comparison with measurements," Proc. SPIE 4976, 107 115 (2003).

8. N. Perlot and D. Fritzsche, "Aperture-averaging: Theory and measurements," Proc. SPIE 5338, 233-242 (2004).

9. L. C. Andrews, "Aperture-averaging factor for optical scintillations of plane and spherical waves in the atmosphere," J. Opt. Soc. Am. A 9, 597-600 (1992).

10. L. C. Andrews, R. L. Phillips, and C. Y. Hopen, Laser Beam Scintil- 
lation with Applications, SPIE Press (2001).

11. N. Perlot, "Turbulence-induced fading probability for a coherent optical communication system through the atmosphere," Appl. Opt. 46, 7218-7226 (2007).

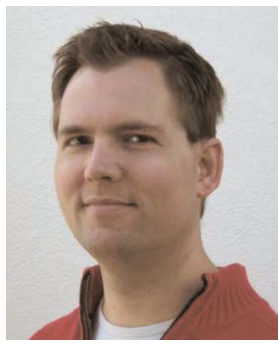

Dirk Giggenbach received his Dipl.-Ing. (M.S.) degree in electrical engineering from the Technical University of Munich in 1994 and the Dr.-Ing. (Ph.D.) degree from the University of the German Federal Armed Forces at Munich in 2004. Since 1995 he has been with the German Aerospace Center (DLR), at Oberpfaffenhofen, Germany where he is head of the Optical free-space Communications Group (OCG). Giggenbach has been involved in several national, and international projects as team leader and project manager. His special research interest is the optical high speed communication through the atmospheric channel. Giggenbach is member of the SPIE.

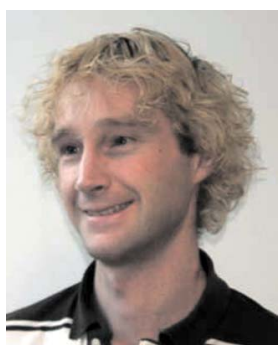

Hennes Henniger received his Dipl.-Ing. degree from the University of Applied Sciences, Munich, in 2002, and his Master of Science degree in 2004. He joined the optical communications group of the Institute for Communications and Navigation in 2001. He was project manager of the mobile near ground optical demonstrator project and work package leader in several other projects. He was developing communication electronics and error correction software for several free-space optical trails. His current research interests are channel modelling and multi layer error protection techniques to overcome fading. Henniger is also serving as a program committee member for the SPIE's conference on free-space laser communications at the Optics \& Photonics symposium in 2008. 